\title{
DETECÇÃO DE BETALACTAMASE DE ESPECTRO ESTENDIDO EM ISOLADOS DE ENTEROBACTÉRIAS PROVENIENTES DE UM HOSPITAL DA REGIÃO DE SANTA TERESA-ES
}

\author{
Thaís Dias Lemos Kaiser ${ }^{1}$ \\ Debora Duarte Santiago ${ }^{2}$ \\ Edson Marques Trindade Mendes $^{3}$ \\ Bruno Vieira Matos ${ }^{4}$
}

KAISER, T. D. L.; SANTIAGO, D. D.; MENDES, E. M. T.; MATOS, B. V. Detecção de betalactamase de espectro estendido em isolados de enterobactérias provenientes de um hospital da região de Santa Teresa-ES. Arq. Cienc. Saúde UNIPAR, Umuarama, v. 20, n. 1, p, 3-7, jan./abr. 2016.

\begin{abstract}
RESUMO: A produção das chamadas Betalactamases de espectro estendido (ESBLs) entre membros da família Enterobacteriaceae tem se tornado comum, sendo cada vez mais identificadas em pacientes hospitalizados, o que representa um importante problema de saúde pública. Detectar a produção das enzimas ESBL em enterobactérias provenientes de diferentes amostras hospitalares originadas de pacientes ambulatoriais e pacientes internados. Foram coletadas 73 amostras, sendo 45 amostras de pacientes internados e 28 de pacientes ambulatoriais de diferentes sítios, e submetidas ao teste fenotípico de disco-aproximação. Do total de amostras, $28,7 \%$ foram produtoras da enzima ESBL. A prevalência da enzima foi maior nos gêneros Escherichia sp. (43\%) e Klebsiella sp. (38\%) sendo a maioria dos isolados provenientes de infecção urinária. Também, $10,7 \%$ dos isolados ambulatoriais foram positivos para detecção da enzima e 5 isolados produtores de ESBL apresentaram sensibilidade no teste de triagem. A prevalência geral de produção de ESBL foi considerável, sendo que a realização de testes fenotípicos é importante para essa detecção. Além disso, a presença dessas bactérias em pacientes ambulatoriais demonstra sua disseminação para fora do ambiente hospitalar.
\end{abstract}

PALAVRAS-CHAVE: Betalactamases. Enterobactérias. ESBL. Infecção. Resistência.

\section{DIAGNOSTIC OF EXTENDED-SPECTRUM BETA-LACTAMASE IN ENTEROBACTERIAL ISOLATES FROM A HOSPITAL IN THE REGION OF SANTA TERESA-ES}

\begin{abstract}
The production of extended-spectrum beta-lactamases (ESBLs) among members of the Enterobacteriaceae family has become common, being increasingly identified in hospitalized patients, which represents a major public health issue. In order to detect the production of ESBL enzymes in enterobacteria originated from different hospital samples originated from outpatients and inpatients, a total of 73 samples were collected, being 45 samples from inpatients and 28 from outpatients from different sites. These samples were submitted to disk-approximation phenotypic testing. From the total sample, 28.7\% produced the ESBL enzyme. The enzyme prevalence was higher in the Escherichia sp. (43\%) and Klebsiella sp. (38\%) genera, with most isolates coming from urinary tract infection. Also, $10.7 \%$ of outpatient isolates were positive for the enzyme detection, with 5 ESBL-producing isolates presenting sensitivity in the screening test. The general prevalence of ESBL production was considerable, since the conduction of phenotypic tests is important for this detection. Furthermore, the presence of these bacteria in outpatients demonstrates its dissemination out of the hospital environment.
\end{abstract}

KEYWORDS: Beta-lactamase. Enterobacteria. ESBL. Infections. Resistance.

\section{Introdução}

As enterobactérias são constituintes normais da microbiota humana, mas podem por vezes, estar associadas a diferentes tipos de infecções, promovendo consideráveis índices de morbidades e mortalidade, principalmente no ambiente hospitalar (INGRAHAM; INGRAHAM, 2011; SOARES, 2013). Nesse contexto, tais bactérias compreendem cerca de $30-35 \%$ de todos isolados de septicemias e mais de $70 \%$ das infecções urinárias, além de também estarem envolvidas em infecções intestinais, e extraintestinais como pneumonias, septicemias neonatais dentre outras. Já em indivíduos da comunidade, as enterobactérias destacam-se como importantes agentes de infecção urinária, e a Escherichia coli, representa cerca de $70 \%$ dos isolados (REIS, 2013; SARMENTO, 2013).

O tratamento de infecções causadas por enterobactérias geralmente é realizado com antimicrobianos da classe dos betalactâmicos, drogas de primeira escolha, devido a sua baixa toxicidade e eficácia terapêutica (GRALHA, 2011).
Contudo, o uso inadequado e em excesso de tais drogas, possibilitou o surgimento de bactérias com diferentes mecanismos de resistência a essa classe de drogas. Sendo a produção de enzimas, denominadas betalactamases, de maior destaque mundial, devido a sua capacidade de hidrolisarem a estrutura dos anéis betalactâmicos, levando a perda da atividade do fármaco. (BRUNTON; CHABNER; KNOLLMANN, 2011; KONG; SCHENEPER; MATHEE, 2010; MARTINS; PICOLI, 2011).

Existem diferentes tipos de betalactamases, e dentre elas, as denominadas Betalactamases de Espectro Estendido (ESBL), produzidas a partir de mutações em genes plasmidiais, tem grande relevância clínica e epidemiológica (MARTINS; PICOLI, 2011). As enzimas ESBL, têm atividade hidrolítica contra penicilinas, cefalosporinas de amplo espectro e monobactâmicos, mas são bloqueadas por compostos conhecidos como inibidores de betalactamases tais como o ácido clavulânico, sulbactam e tazobactam. Todavia, a localização plasmidial desse gene de resistência facilita a sua disseminação entre os Gram negativos, principalmente entre

DOI: https://doi.org/10.25110/arqsaude.v20i1.2016.5257

${ }^{1}$ Farmacêutica Bioquímica. Endereço: Rua Milton Caldeira, Praia de Itapuã, Vila Velha ES. E-mail tdlkaiser@gmail.com

${ }^{2}$ Farmacêutica. Endereço: Rua Coste e Silva, № 517, Bairro: Bela vista. Cidade: Aracruz-ES. E-mail: deboraduartesantiago@gmail.com

${ }^{3}$ Farmacêutico. Endereço: Rua Tarson Paiva, Nº 8, Bairro: Alto Dona Augusta. Cidade: Cariacica-ES. E-mail: edson_marques07@hotmail.com

${ }^{4}$ Farmacêutico. Endereço: Rua Jacinto Antonio. Bairro: Centro, Cidade: Ecoporanga-ES. E-mail: brunovieiramatos@gmail.com 
as enterobactérias, por meio do processo de conjugação. Esses plasmídeos de resistência muitas vezes já possuem genes que conferem resistência a outras classes de drogas como as quinolonas e os aminoglicosídeos e por isso, cepas produtoras de ESBL isoladas principalmente no ambiente hospitalar, tendem a ser multirresistentes (LAGO; FUENTEFRIA; FUENTEFRIA, 2010; MARTINS, 2012; SÁ, 2010).

O diagnóstico adequado ainda tem sido um grande obstáculo aos microbiologistas e estudiosos, devido ao fato de existirem uma variedade de fenótipos da enzima ESBL, que conferem afinidade da enzima a uma ou outra droga alvo. Além disso pode ainda apresentar uma sensibilidade à determinada classe de betalactâmico em específico (SOARES, 2013). Sabe-se que é fundamental conhecer o comportamento das enterobactérias produtoras das betalactamases, principalmente no ambiente hospitalar. E a não identificação de tais cepas no laboratório de microbiologia, pode levar ao uso inadequado de antimicrobianos e consequentemente a falhas nas medidas de controle das infecções relacionadas à assistência à saúde, contribuindo assim para o aumento da transmissão de microorganismos multirresistentes (MARTINS; PICOLI, 2011).

\section{Material e Métodos}

\section{Localização do estudo e amostras avaliadas}

Este estudo foi submetido ao comitê de ética em Pesquisa do Hospital Meridional S/A e aprovado sob. o número de CAAE 1651013.3.0000.5070, em agosto de 2013.

Foram avaliados 73 isolados de enterobactérias provenientes de diferentes amostras biológicas de pacientes atendidos em um Hospital localizado na cidade de Santa Teresa, Espírito Santo. Foram incluídas amostras originadas de pacientes externos (ambulatoriais) e pacientes internados nesse hospital, no período de Julho de 2013 até Outubro de 2014.

Os isolados bacterianos, provenientes de diferentes amostras biológicas foram identificados, e tiveram o seu perfil no antibiograma realizado previamente pela equipe do Laboratório de Análises Clínicas do Hospital. Posteriormente, os resultados foram disponibilizados para esse estudo e os microorganismos isolados foram selecionados para a realização do teste fenotípico de pesquisa da enzima, no Laboratório de Microbiologia da Escola Superior São Francisco de Assis.

\section{Processamento dos isolados}

No laboratório, os isolados bacterianos recebidos foram repicados em meio de cultura Ágar sangue para a recuperação da amostra e confirmação da pureza. Posteriormente cada uma delas foi submetida ao Teste fenotípico de Disco-aproximação (JARLIER et al., 1988) para a pesquisa fenotípica da produção da enzima ESBL.

\section{Teste de disco-aproximação}

O método é baseado na característica dessa enzima de apresentar sensibilidade aos inibidores de betalactamases. A detecção da enzima ESBL foi feita mediante a atividade sinérgica de discos de betalactâmicos com e sem o inibidor de betalactamases, foi utilizado o ácido clavulânico como inibidor (JARLIER et al., 1988). Foi feita uma suspensão, ajustada à concentração 0,5 na escala de MacFarland, em salina estéril para cada isolado. Em seguida, foram submetidas à semeadura com auxílio de um swab estéril na superfície do meio Ágar Mueller-Hinton (HIMEDIA). Após 10 minutos os discos de amoxicilina/clavulanato $(30 \mu \mathrm{g})$, ceftriaxona $(30 \mu \mathrm{g})$, ceftazidima $(30 \mu \mathrm{g})$, cefepime $(30 \mu \mathrm{g})$ e aztreonam $(30 \mu \mathrm{g})($ CEFAR), foram colocados a uma distância de $25 \mathrm{~mm}$ entre eles, para se difundirem pelo meio de cultura promovendo um efeito inibitório no crescimento. As amostras foram incubadas por $18-24 \mathrm{~h}$ em estufas a $35 \pm 2^{\circ} \mathrm{C}$. $\mathrm{O}$ resultado positivo foi identificado pela extensão do halo de inibição entre o disco de amoxicilina/clavulanato e os demais discos sem inibidor, ou pelo o aparecimento de uma "zona fantasma", sem crescimento microbiano entre esses. Como controles foram utilizados as cepas de $K$. pneumonia e ATCC 700603 (produtora de ESBL) e E. coli ATCC 25922 (não produtora de ESBL).

\section{Resultados}

Foram avaliados 73 isolados bacterianos nesse estudo, sendo 45 provenientes de pacientes internos e 28 de pacientes ambulatoriais atendidos no hospital. A tabela 1 mostra a distribuição dos isolados conforme espécime clínico bem como a origem do paciente.

Tabela 1: Distribuição dos isolados de acordo com o sítio de infecção e origem do paciente

\begin{tabular}{|c|c|c|c|c|c|c|}
\hline \multirow{2}{*}{ Espécies } & \multicolumn{4}{|c|}{ Pacientes internados n(\%) } & \multirow{2}{*}{$\begin{array}{c}\text { Pacientes } \\
\text { ambulatoriais } \mathbf{n}(\mathbf{5}) \\
\text { Urina }\end{array}$} & \multirow{2}{*}{ Total n(\%) } \\
\hline & Urina & Swabs de Ferida & Secreção & Sangue & & \\
\hline Escherichia coli & $27(37 \%)$ & $1(1,4 \%)$ & - & - & $25(34,2 \%)$ & $53(72,6 \%)$ \\
\hline Klebsiella pneumoniae & $6(8,2 \%)$ & $2(2,7 \%)$ & - & - & $2(2,7 \%)$ & $10(13,7 \%)$ \\
\hline Klebsiella ozanae & $1(1,4 \%)$ & - & - & - & - & $1(1,4 \%)$ \\
\hline Proteus mirabilis & $2(2,7 \%)$ & - & - & - & $1(1,4 \%)$ & $3(4,1 \%)$ \\
\hline Enterobacter aerogenes & - & $2(2,7 \%)$ & - & - & - & $2(2,7 \%)$ \\
\hline Enterobacter gergoviae & - & $2(2,7 \%)$ & - & - & - & $2(2,7 \%)$ \\
\hline Enterobacter sp. & - & - & $1(1,4 \%)$ & $1(1,4 \%)$ & - & $2(2,7 \%)$ \\
\hline Total & $36(49, \%)$ & $7(9,5 \%)$ & $1(1,4 \%)$ & $1(1,4 \%)$ & $28(38, \%)$ & $73(100 \%)$ \\
\hline
\end{tabular}


Quanto à detecção fenotípica de ESBL, das 73 amostras isoladas, $21(28,7 \%)$ apresentaram um resultado positivo para a produção de ESBL, sendo a grande maioria dessas provenientes de pacientes internados e um pequeno percentual de pacientes ambulatoriais. A tabela 2 apresenta a distribuição da prevalência da enzima ESBL de acordo com a origem do paciente.

Tabela 2: Distribuição dos isolados produtores de ESBL de acordo com a origem do paciente

\begin{tabular}{lcc}
\hline \multicolumn{1}{c}{ Pacientes } & Total de isolados & $\begin{array}{c}\text { Total de amostras } \\
\text { Positivas n (\%) }\end{array}$ \\
\hline Internados & 45 & $18(40 \%)$ \\
Ambulatoriais & 28 & $3(10,7 \%)$ \\
Total & 73 & $21(28,7 \%)$ \\
\hline
\end{tabular}

A figura 1 exibe a prevalência de produção da enzima conforme espécie e sítio de isolamento. Dessas 21 amostras positivas a maioria foi $E$. coli e $K$. pneumoniae isolados de amostra de urina.
Figura 1: Espécies produtoras de ESBL no teste fenotipico e sitio de isolamento
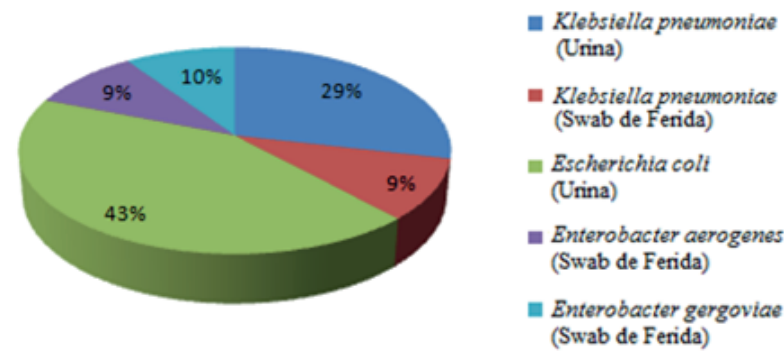

Na tabela 3 está apresentada à correlação do perfil de sensibilidade das amostras no teste de triagem pelo antibiograma feito no Laboratório Madre Regina com os resultados obtidos do teste fenotípico para ESBL. Nota-se que das 21 amostras positivas para ESBL, 5 apresentaram sensibilidade nos discos de triagem do antibiograma.

Tabela 3: Comparação do resultado da triagem para os discos no antibiograma com o teste para ESBL

\begin{tabular}{ccccc}
\hline $\begin{array}{c}\text { Quantidade amostras } \\
\mathbf{n}\end{array}$ & Ceftriaxona & Ceftazidima & Cefepime & Teste ESBL \\
\hline 47 & Sensível & Sensível & Sensível & Negativo \\
12 & Resistente & Resistente & Resistente & Positivo \\
1 & Resistente & Sensível & Resistente & Positivo \\
5 & Sensível & Sensível & Sensível & Positivo \\
2 & Resistente & Resistente & Sensível & Positivo \\
1 & Resistente & Sensível & Sensível & Positivo \\
2 & NT* & Sensível & Sensível & Negativo \\
2 & Senstível & NT* & NT* & Negativo \\
\hline
\end{tabular}

*NT. Não Testado

\section{Discussão}

A produção de ESBL por enterobactérias tem um importante impacto nas taxas de morbidade e até mortalidade bem como nos custos de tratamento hospitalar e comunitário (SOARES, 2013). No presente estudo foi encontrada uma prevalência considerável de produção da enzima ESBL entre os isolados avaliados $(28,7 \%)$. Outros trabalhos também apresentaram prevalências aproximadas, como Lenhard-Vidal e colaboradores (2011), que estudaram a prevalência de ESBL entre enterobactérias em um hospital público de Maringá- PR, Brasil, e obteve uma prevalência geral de $22 \%$. Bem como Lago, Fuentefria e Fuentefria (2010), que estudaram amostras isoladas de pacientes internados no hospital São Vicente de Paulo em Passo Fundo- RS, Brasil, e encontraram uma prevalência de $24,8 \%$.

As espécies bacterianas de maior a prevalência da enzima foram E. coli (43\%) e Klebsiella pneumoniae (38\%) em comparação com os demais gêneros isolados. Apesar de esse resultado poder ser um reflexo do grande número de isolados pertencentes a essas espécies neste trabalho, na literatura, é descrito, desde o primeiro relato, que essas duas espécies são as mais comuns identificadas como produtoras de ESBL (COSTA, 2013; GRALHA, 2011; MANA, 2014). Contudo, muitos trabalhos têm relatado o isolamento de outros gêneros de enterobactérias também capazes de produzir ESBL (LAGO; FUENTEFRIA; FUENTEFRIA, 2010; LENHARD-VIDAL, 2011). Também encontramos isolados de Enterobacter sp. produtores de ESBL. A presença da enzima em várias outras espécies evidencia a facilidade de transmissão dessa resistência para outros membros desta família.

A presença de bactérias multirresistentes no ambiente hospitalar é comum, principalmente por ser um ambiente onde a pressão seletiva é grande devido ao uso constante de antimicrobianos, e isso é demonstrado em vários estudos (ABREU et al., 2011; REIS, 2013; SARMENTO, 2013). Contudo, a pesquisa de bactérias resistentes na comunidade tem crescido como maneira de mostrar a disseminação de tais bactérias para fora do ambiente hospitalar. Neste trabalho dentre os pacientes ambulatoriais a prevalência da produção de ESBL foi de 10,7\%. Abreu e colaboradores em 2012 realizaram um estudo em São Luiz, Brasil, com 472 amostras de enterobactérias de infecções do trato urinário adquiridas na comunidade, no qual 36 foram positivas para produção de ESBL, obtendo um percentual de 7,6\%. Em outro estudo realizado em uma comunidade de Juiz de Fora, 
Brasil, a prevalência foi de $3 \%$ da enzima ESBL, sendo $K$. pneumoniae e E. coli as principais produtoras (WOLLHEIM, C. et al., 2011).

Diferentes fatores podem contribuir para a presença de resistência bacteriana fora do ambiente hospitalar, tais como o uso indiscriminado de antimicrobianos também pela comunidade, a descontinuidade do tratamento pelos pacientes ou esquecimentos durante o tratamento, prescrição médica empírica sem uma análise microbiológica anterior a respeito do agente etiológico e do perfil de resistência, falta de qualificação de profissionais de saúde ou até circulação de pacientes entre unidades de saúde (SÁ, 2010; SARMENTO, 2013).

A produção de ESBL em enterobactérias é clinicamente relevante sendo uma importante causa de falha no tratamento da infecção quando há uso de cefalosporinas, mesmo quando esses microorganismos parecem ser sensíveis no antibiograma de rotina do laboratório. Ao comparar a sensibilidade dessas bactérias no antibiograma com o resultado do teste para ESBL, houve variação quanto a resistência a uma ou outra cefalosporina. Isso acontece devido existência de uma variedade de genótipos para ESBL, sendo a maior parte derivada das enzimas TEM e SHV e CTX-M muito comuns principalmente em E.coli e Klebsiella pneumoniae. Essas enzimas e suas variantes conferem cada uma diferentes afinidades na hidrólise de betalactâmicos como as penicilinas e as cefalosporinas, podendo refletir no antibiograma com cepas que apresentam sensibilidade a uma cefalosporina de $3^{\mathrm{a}}$ e resistência a uma outra (SÁ, 2010; SOARES, 2013).

Analisando a tabela 3, foi possível observar que 5 amostras positivas para o teste de detecção de ESBL, foram sensíveis a todas cefalosporinas no teste de triagem. Tal resultado demonstra a importância e a necessidade da realização de testes adicionais ao antibiograma na detecção desses mecanismos de resistência, que contribuirão para o diagnóstico adequado, orientando assim a escolha da terapia antimicrobiana. Ainda, pode ser observado que 8 amostras produtoras para ESBL apresentaram sensibilidade ao cefepime no antibiograma. O Cefepime é uma cefalosporina de $4^{\mathrm{a}}$ geração, com amplo espectro de ação e tem sido muito indicado no tratamento de infecções de Enterobactérias, pois tem uma rápida penetração por meio da membrana exterior da célula. Um estudo realizado no Hospital da Universidade Federal do Paraná avaliou por diferentes métodos a sensibilidade de 280 cepas de enterobactérias produtoras de ESBL, ao cefepime, e 35 (12.1\%) foram sensíveis com CMI (Concentração Mínima Inibitória) $\leq 8 \mathrm{mcg} / \mathrm{ml}$ (NOGUEIRA et al., 2011). Já Mudshingkar e colaboradores (2014) encontraram sensibilidade ao cefepime em 65,4\% das 219 amostras de enterobactérias produtoras de ESBL em seu estudo. A escolha dessa droga como opção no tratamento de ESBL reduz o uso de carbapenemas em tais infecções, minimizando a seleção de cepas resistentes. Entretanto, apesar de ser uma opção, seu uso no tratamento de infecções causadas por cepas produtoras de ESBL traz controvérsias pois tem sido observada falha terapêutica em pacientes graves infectados com ESBL (CHOPRA et al., 2012; LEE et al., 2013; BLANDINO et al., 2014).

\section{Conclusão}

Em nosso trabalho, a prevalência encontrada reflete uma realidade observada em diferentes regiões do país. Contudo, a presença da enzima em outros membros da família Enterobacteriaceae mostra a capacidade de disseminação da enzima entre diferentes espécies de enterobactérias. Ainda, a detecção de ESBL em isolados de pacientes da comunidade, mostra a capacidade de disseminação desses microorganismos também para fora do ambiente hospitalar.

Além disso, a presença de alguns isolados produtores de ESBL detectados pelo teste fenotípico, porém com sensibilidade no teste rotineiro de triagem do laboratório pelo antibiograma demonstram a necessidade do uso de métodos adicionais, além do antibiograma que auxiliem na detecção adequada destes mecanismos de resistência.

Medidas na contenção da disseminação dessa resistência devem ser tomadas e para isso, a detecção precoce desses mecanismos pelo laboratório, pode auxiliar no monitoramento da resistência antimicrobiana, evitar falhas na terapia e até mesmo direcionar as estratégias de intervenção e controle.

\section{Referências}

ABREU, A. G. et al. Extended-spectrum -lactamaseproducing enterobacteriaceae incommunity-acquired urinary tract infections in São Luís, Brazil. Brazilian Journal of Microbiology, v. 44, n. 2, p. 469-471, 2012. Disponível em: $<$ http://www.ncbi.nlm.nih.gov/pmc/articles/PMC3833145/ pdf/bjm-44-469.pdf>. Acesso em: 10 nov. 2014.

ABREU, A. G. et al. Nosocomial infection and characterization of extended-spectrum $\beta$-lactamasesproducing Enterobacteriaceae in northeast Brazil. Revista da Sociedade Brasileira de Medicina Tropical, v. 44, n. 4, p. 441-446, 2011. Disponível em: <http://www.scielo.br/ pdf/rsbmt/v44n4/08.pdf $>$. Acesso em: 10 nov. 2014.

BLANDINO, G. et al. Antimicrobial susceptibility of strains of Enterobacteriaceae isolated from bloodstream infections using current CLSI and EUCAST breakpoints. ScientificResearch, v. 6, n. 2, p. 153-157, 2014. Disponível em: <http:/www.scirp.org/journal/PaperInformation. aspx?PaperID=42478> . Acesso em: 15 nov. 2014.

BRUNTON, L. L.; CHABNER, B. A.; KNOLLMANN B. C. As bases farmacológicas da terapêutica de Goodman e Gilman. 12. ed. São Paulo: Artmed, 2011. p. 2112.

CHOPRA, T. et al. Impact of cefepime therapy on mortality among patients with bloodstream infections caused by extended-spectrum-b-lactamase-producing Klebsiella pneumoniae and Escherichia coli. Antimicrob Agents Chemother, v. 56, p. 3936-3942, 2012.

COSTA, S. I. A. D. Disseminação horizontal de genes que codificam para betalactamases de espectro alargado em isolados de Enterobacteriaceae de origem hospitalar. 2013. Tese (Doutorado em Ciências Farmacêuticas) Faculdade de Ciências da Saúde, Universidade Fernando 
Pessoa, Portugal, 2013.

GRALHA, R. E. F. Métodos de pesquisa de betalactamases em amostras clínicas - estudo de revisão. 2011. Tese (Mestre em Ciências Farmacêuticas) - Faculdade de Ciências da Saúde, Universidade Fernando Pessoa. Portugal, 2011.

INGRAHAM, J. L.; INGRAHAM, C. A. Introdução à microbiologia: uma abordagem baseada em estudos de casos. 3. ed. São Paulo: Cengage Learning, 2011. p. 776.

JARLIER, V. et al. Extended broadspectrum $\beta$-lactamases conferring transferable resistance to newer $\beta$-lactam agents in Enterobacteriaceae: hospital prevalence and susceptibility patterns. Rev. Infect. Dis. v. 10, p. 867-878, 1988

KONG, K. F.; SCHENEPER, L.; MATHEE, K. Beta-lactam antibiotics: from antibiosis to resistance and bacteriology. APMIS, v. 118, n.1, p. 1-36, 2010. Disponível em: <http:// www.ncbi.nlm.nih.gov/pmc/articles/PMC2894812/pdf/ nihms156704.pdf>. Acesso em: 28 set. 2014.

LAGO, A.; FUENTEFRIA, S. R.; FUENTEFRIA, D. B. Enterobactérias produtoras de ESBL em Passo Fundo, Estado do Rio Grande do Sul, Brasil. Revista da Sociedade Brasileira de Medicina Tropical, v. 43, n. 4, p. 430-444, 2010. Disponível em: < http://www.scielo.br/pdf/rsbmt/ v43n4/a19v43n4.pdf>. Acesso em: 11 out. 2014.

LEE, N.Y et al. Cefepime therapy for monomicrobial bacteremia caused by cefepime-susceptible extendedspectrum Beta-Lactamase-producing enterobacteriaceae: MIC Matters. Clin Infect Dis. v. 56, p. 488-495, 2013.

LENHARD-VIDAL, A. High prevalence rate of extended-spectrum beta-lactamases (ESBL) among Enterobacteriaceae in a small Brazilian public hospital. Brazilian Journal of Pharmaceutical Sciences, v. 47, n. 4, p. 701-707, 2011. Disponível em: <http://www.scielo.br/ pdf/bjps/v47n4/06.pdf>. Acesso em: 06 nov. 2014.

MANA, M. et al. Prevalência de Klebsiella spp. ESBL isolada em Hospital Escola do Sul de Minas Gerais. Revista da Universidade Vale do Rio Verde, v.12, n. 2, p. 497-506, 2014. Disponível em: $<$ http://revistas.unincor.br/index.php/ revistaunincor/article/view/1503/pdf_229>. Acesso em: 16 maio 2015.

MARTINS, A. C.; PICOLI, S. U. Métodos alternativos para detecção de betalactamase de espectro estendido em Escherichia coli e Klebsiellapneumoniae. Jornal Brasileiro de Patologia e Medicina Laboratorial, v. 47, n. 4, p. 421-426, 2011. Disponível em: <http://www.scielo.br/ pdf/jbpml/v47n4/v47n4a05>. Acesso em: 28 set. 2014.

MARTINS, A. F. M. M. Prevalência de resistência a antimicrobianos em isolados ambientais de Escherichia coli e enterococos. 2012. Tese (Doutorado em Tecnologia Bioquímica em Saúde)- Instituto Politécnico do Porto,
Escola Superior de Tecnologia da Saúde do Porto, Porto, Portugal, 2012.

MUDSHINGKAR, S. S. et al. Cefepime/tazobactam-a promising BL-BLI combination against multidrug resistant Gram negative bacteria. International J. of Healthcare and Biomedical Research, v. 2, n. 3, p. 127-128, 2014 Disponível em: <http://ijhbr.com/pdf/4\%202014\%20127128.pdf $>$. Acesso em: 15 nov. 2014.

NOGUEIRA, K. S. et al. Cefepime versus extendedspectrum $\beta$-lactamaseproducing Enterobacteriaceae. Brazilian Journal of Infectious Diseases, v.15, n. 2, p. 167-169, 2011. Disponível em: $<$ http://www.scielo.br/pdf/bjid/v15n2/v15n2a14.pdf $>$. Acesso em: 14 nov. 2014.

REIS, H. P. L. C. et al. Avaliação da resistência microbiana em hospitais privados de Fortaleza - Ceará. Revista Brasileira de Farmácia, v. 94, n. 1, p. 83-87, 2013. Disponível em: <http://www.rbfarma.org.br/files/rbf-201394-1-13.pdf>. Acesso em: 28 set. 2014.

SÁ, C. M. F. ESBL em enterobactérias no Centro Hospitalar Póvoa de Varzim/Vila do Conde. 2010. Tese (Doutorado em Microbiologia) - Departamento de Biologia, Universidade de Aveiro, Portugal, 2010.

SARMENTO, L. B. S. Incidência de infecções hospitalares por enterobactérias em um Hospital de Campina Grande-PB. 2013. Tese (Doutorado em Farmácia) - Departamento de Farmácia, Universidade Estadual da Paraíba, Campina Grande, 2013.

SOARES, S. F. Epidemiologia de estirpes produtoras de ESBL em ITU. 2013. Tese (Doutorado em Bioquímica) - Departamento de Química, Universidade de Aveiro, Portugal, 2013.

WOLLHEIM, C. et al. Nosocomial and community infections due to class A extended-spectrum b-lactamase (esbla)-producing Escherichia coli and Klebsiella spp. in southern Brazil. Brazilian Journal of Infectious Diseases, v. 15, n. 2, p. 138-143, 2011. Disponível em: <http://www. scielo.br/pdf/bjid/v15n2/v15n2a08.pdf >. Acesso em: 10 nov. 2014

Recebido: 06/08/2015 Aceito: $17 / 02 / 2016$ 